\title{
Kovacs Memory Effect with an Optically Levitated Nanoparticle
}

\author{
Andrei Militaru $\odot,{ }^{1}$ Antonio Lasanta $\odot,{ }^{2,3,4,5}$ Martin Frimmer, ${ }^{1}$ Luis L. Bonilla $\odot,{ }^{6,4,7}$ \\ Lukas Novotny, ${ }^{1, \dagger}$ and Raúl A. Rica $\oplus^{5,8, *}$ \\ ${ }^{1}$ Photonics Laboratory, ETH Zürich, CH-8093 Zürich, Switzerland \\ ${ }^{2}$ Departamento de Álgebra, Facultad de Educación, Economía y Tecnología de Ceuta, \\ Universidad de Granada, Cortadura del Valle, s/n, 51001 Ceuta, Spain \\ ${ }^{3}$ Grupo de Teorías de Campos y Física Estadística, Instituto Gregorio Millán, Universidad Carlos III de Madrid, \\ Unidad Asociada al Instituto de Estructura de la Materia, CSIC, Spain \\ ${ }^{4}$ Grupo de Matemática Aplicada a la Física de la Materia Condensada, Instituto Gregorio Millán, \\ Universidad Carlos III de Madrid, Unidad Asociada al Instituto de Ciencias de Materiales de Madrid, CSIC, Spain \\ ${ }^{5}$ Nanoparticles Trapping Laboratory, Universidad de Granada, 18071 Granada, Spain \\ ${ }^{6}$ Departamento de Matemáticas, Universidad Carlos III de Madrid, 28911 Leganés, Spain \\ ${ }^{7}$ Instituto Gregorio Millán, Universidad Carlos III de Madrid, 28911 Leganés, Spain \\ ${ }^{8}$ Universidad de Granada, Department of Applied Physics and Research Unit "Modeling Nature” (MNat), \\ 18071 Granada, Spain
}

(Received 8 April 2021; accepted 12 August 2021; published 24 September 2021)

\begin{abstract}
The understanding of the dynamics of nonequilibrium cooling and heating processes at the nanoscale is still an open problem. These processes can follow surprising relaxation paths due to, e.g., memory effects, which significantly alter the expected equilibration routes. The Kovacs effect can take place when a thermalization process is suddenly interrupted by a change of the bath temperature, leading to a nonmonotonic evolution of the energy of the system. Here, we demonstrate that the Kovacs effect can be observed in the thermalization of the center of mass motion of a levitated nanoparticle. The temperature is controlled during the experiment through an external source of white Gaussian noise that mimics an effective thermal bath at a temperature that can be changed faster than any relaxation time of the system. We describe our experiments in terms of the dynamics of a Brownian particle in a harmonic trap without any fitting parameter, suggesting that the Kovacs effect can appear in a large variety of systems.
\end{abstract}

DOI: 10.1103/PhysRevLett.127.130603

Memory effects (MEs) are a unique feature of nonequilibrium systems. The appearance of memory involves the influence of the past history of a system on its present state and can critically affect its evolution [1]. Most MEs appear as the transient evolution from a particular choice of initial conditions or as a response to a perturbation before the system relaxes to the equilibrium or stationary state. They are ubiquitous in science, appearing in almost every field, including physics, chemistry, biology, and materials science. MEs have been intensively studied in prototypical outof-equilibrium systems, such as disordered media [2-4], active matter [5], and polymers [6].

Thermalization processes are of utmost importance in many technological applications and are prone to feature MEs. As an example, the counterintuitive Mpemba effect has been recently rediscovered and attracted significant attention [7]. When the Mpemba effect takes place, the hotter of two systems can be cooled faster (or the cooler of two systems can be heated faster). This effect has been found in Markovian systems [8,9], granular matter [10,11], spin glasses [12], water [13], the quantum Ising spin model [14], and very recently in a generalization to Markovian open quantum systems [15]. Under different conditions, the
Kovacs effect (KE) [16,17] appears when trying to accelerate the cooling with a more elaborated protocol. Consider a system that at time $t=0$ is in equilibrium with a hot thermal reservoir at temperature $T_{H}$ and that needs to be cooled down quickly to a warm temperature $T_{W}<T_{H}$. The Kovacs protocol first quenches the system by setting a cold temperature $T_{C}<T_{W}$ and then applies the target temperature $T_{W}$ at a later time $t_{W}$, when the system's temperature is already $T_{W}$. Intuition tells us that with the Kovacs protocol the system reaches $T_{W}$ faster than in a one-step process. What actually happens, however, is that the system's temperature may not remain at $T_{W}$ : it may instead evolve following a nonmonotonic path that features a hump.

$\mathrm{KE}$ and analogous crossover effects have been theoretically predicted in a large number of models, including spin glasses [2], glasses [18], granular matter [19,20], active matter [21], molecular gases [22] and it has been generalized to athermal systems [23]. As far as we know, however, the experimental observations are scarce. Apart from the original experiment with polymers $[16,17]$, the KE has been observed only in ferroelectrics [24] and granular matter [25]. 
An optically trapped particle is an outstanding platform to experimentally explore nonequilibrium dynamics at the microscale [26,27]. MEs due to the inertia of the fluid have been previously observed with microparticles dispersed in a liquid, both freely diffusing [28] and trapped with optical tweezers [29,30]. Recent studies have engineered optimum protocols to speed up the thermalization process by taking advantage of the Mpemba effect [31] and of the system's transient response upon a sudden change of a control parameter [32,33]. In this Letter, we demonstrate both experimentally and theoretically that the $\mathrm{KE}$ can be observed using an optically levitated nanoparticle. We show that our results can be explained by the interplay of the different equilibration timescales predicted by the dynamics of a Brownian particle in a harmonic trap with intermediate damping, i.e., in the transition from the overto underdamped regime. This regime, which has been shown to feature very interesting phenomena, such as the recently observed Kramers turnover [34], remains to date largely unexplored.

Our experimental setup is shown in Fig. 1(a). We create an optical potential for a charged silica nanoparticle (radius $\approx 90 \mathrm{~nm}$ ) by focusing an $x$-polarized laser beam traveling along the $z$ axis through a 0.8 NA microscope objective.

(a)
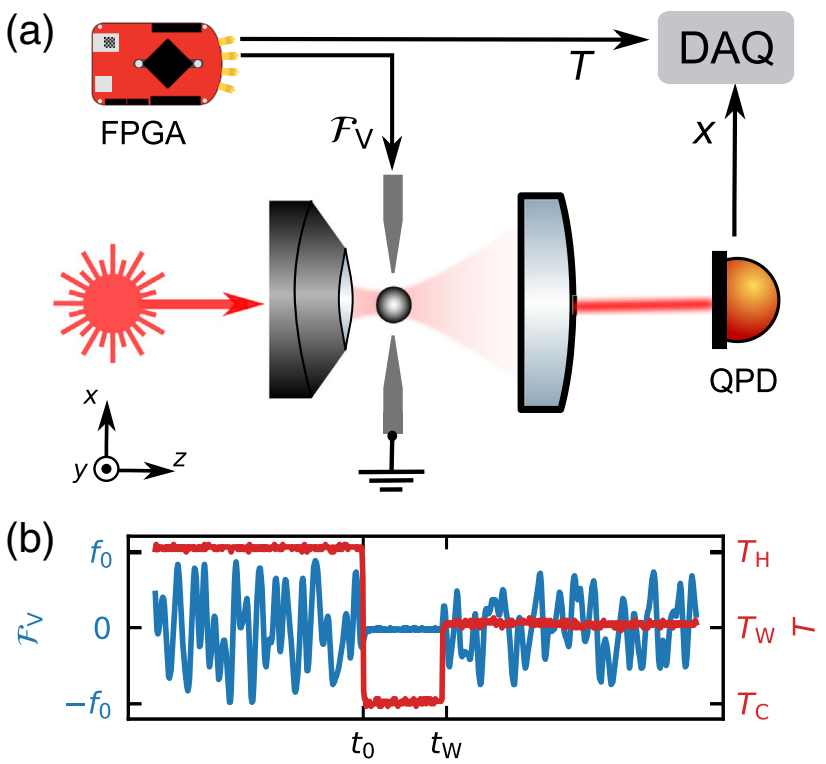

FIG. 1. (a) A $1064 \mathrm{~nm}$ wavelength laser is focused through a microscope objective to create an optical trap for a charged silica nanoparticle. We use homodyne detection to record the particle's position with a quadrant photodiode (QPD). We use a custom programmed FPGA to tune the effective temperature of the particle along the $x$ axis by applying a white Gaussian electrostatic force. The instantaneous temperature $T$ is stored together with the position signal by a data acquisition card (DAQ). (b) Force $\mathcal{F}_{v}$ applied on the particle by the FPGA and corresponding temperature $T$ during the Kovacs protocol shown in Fig. 2(b). $f_{0}$ is a reference force in arbitrary units.
We collect the trapping and the scattered light with a collection lens and apply a standard homodyne detection scheme to measure the position of the particle $[35,36]$. We use a pair of electrodes mounted along the $x$ axis to control the motion of the particle through an externally applied electrostatic force [37,38]. To first order, the motion of the particle along the $x$ axis obeys the following equation:

$$
m \ddot{x}+m \Gamma \dot{x}+m \Omega_{0}^{2} x=\mathcal{F}_{\text {th }}+\mathcal{F}_{v},
$$

with analogous expressions for the other two axes $y$ and $z$. In Eq. (1), $m \approx 6.6 \mathrm{fg}$ is the mass of the particle, $\Omega_{0}=$ $2 \pi \times 159(1) \mathrm{kHz}$ is the resonance frequency of the optical trap along the $x$ axis, and $\Gamma=2 \pi \times 399(2) \mathrm{kHz}$ is the damping rate due to collisions with gas molecules at ambient pressure (around 1 bar). By virtue of the fluctuationdissipation theorem, the damping $\Gamma$ leads to a fluctuating force $\mathcal{F}_{\text {th }}$ with $\left\langle\mathcal{F}_{\text {th }}(t)\right\rangle=0$ and $\left\langle\mathcal{F}_{\text {th }}(t) \mathcal{F}_{\text {th }}(t+\tau)\right\rangle=$ $2 m k_{B} T_{C} \Gamma \delta(\tau)$, where $T_{C}=298 \mathrm{~K}$ is the laboratory temperature [39]. Throughout this Letter, the notation $\langle\cdot\rangle$ refers to the ensemble average and not to a time average. Note that our system lives in the largely unexplored damping regime that is neither underdamped $\left(\Gamma<2 \Omega_{0}\right)$ nor deeply overdamped $\left(\Gamma \gg \Omega_{0}\right)$. By adding an external stochastic force $\mathcal{F}_{v}$, we increase the strength of the fluctuation without affecting the dissipation, such that the net effect is to tune the effective temperature of the c.m. motion [37]. The c.m. temperature is controlled in time by a custom programmed field programmable gate array (FPGA) that tunes the variance of $\mathcal{F}_{v}$, as shown in Fig. 1(b). The bandwidth of $\mathcal{F}_{v}$ is $5 \mathrm{MHz}$, such that it can be considered a white Gaussian force for all purposes (see Fig. S2 in the Supplemental Material [40]). In the rest of this Letter, we consider three values of the c.m. temperature: (i) cold temperature $T_{C}=298 \mathrm{~K}$ (when $\mathcal{F}_{v}=0$ ), (ii) warm temperature $T_{W}=1290 \mathrm{~K}$, and (iii) hot temperature $T_{H}=2450 \mathrm{~K}$.

The thermodynamic quantity of interest is the particle's potential energy, defined as $\langle U\rangle=m \Omega_{0}^{2}\left\langle x^{2}\right\rangle / 2$. We measure the time evolution of $\langle U\rangle$ by computing the ensemble average over 10000 trajectories of the particle that start from the same equilibrium state. Figure 2 displays the evolution of the potential energy and the corresponding changes in effective temperature for three different protocols.

The first protocol is the equilibration from the hot to the cold temperature (light blue triangles). This quenching of the effective temperature from $T_{H}$ to $T_{C}$ induces an equilibration of the potential energy of the particle that takes about $4 \mu \mathrm{s}$. Interestingly, rather than decaying exponentially toward $k_{B} T_{C} / 2$, the potential energy remains at $k_{B} T_{H} / 2$ for a duration of $1 / \Gamma \approx 400 \mathrm{~ns}$ before decaying. In general, the potential energy of an underdamped (overdamped) oscillator would follow a simple exponential decay with rate given by $\Gamma\left(\Omega_{c}=\Omega_{0}^{2} / \Gamma\right)[31,43]$. 


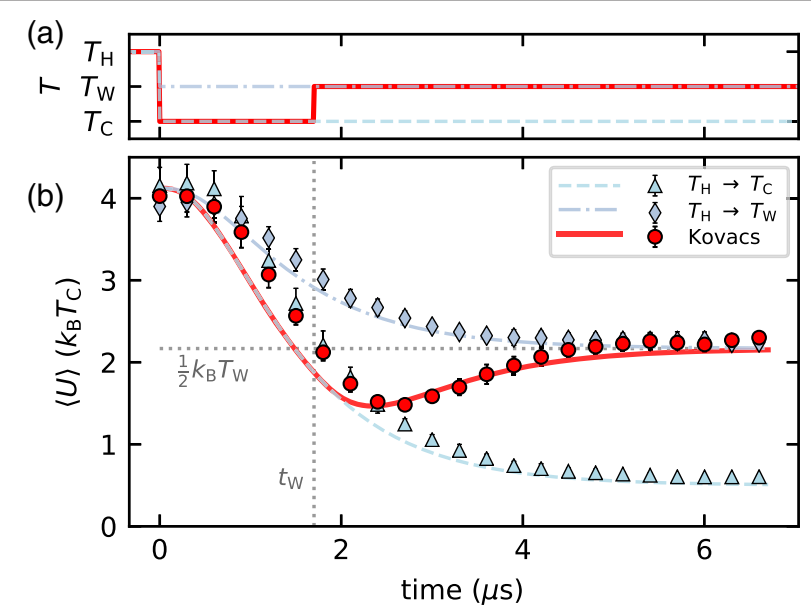

FIG. 2. (a) Temperature of the effective bath during the cooling protocol $T_{H} \rightarrow T_{C}$ (dashed light blue line), cooling protocol $T_{H} \rightarrow T_{W}$ (dash-dotted silver line), and Kovacs protocol (red solid line). (b) Average potential energy during the protocols described in (a). Discrete symbols (light blue triangles, silver diamonds, and red circles) are used for experimental data, whereas lines are used for the theoretical curves. The horizontal dotted line is the equilibrium value $k_{B} T_{W} / 2$ related to the warm temperature. The vertical dotted line signals the switch time between $T_{C}$ and $T_{W}$ for the Kovacs protocol. The estimation of the error bars is described in [40].

The nonexponential equilibration of $\langle U\rangle$ for our intermediate damping regime suggests that more than one timescale is involved in the particle's equilibration.

The second protocol we analyze is the equilibration between hot and warm temperature (silver diamonds). The $T_{H} \rightarrow T_{W}$ equilibration exhibits the same qualitative behavior of $T_{H} \rightarrow T_{C}$ except for the final state, which in this case is $k_{B} T_{W} / 2$. During the $T_{H} \rightarrow T_{C}$ equilibration, the intermediate value of $k_{B} T_{W} / 2$ is reached after a time $t_{W}=1.7 \mu \mathrm{s}$.

Finally, we apply the Kovacs protocol (red circles in Fig 2): (i) we let the system equilibrate at $T_{H}$, (ii) at time $t=0$ we change the temperature to $T_{C}$, and (iii) at time $t_{W}$ we switch the temperature to $T_{W}[20,44,45]$. Despite the fact that $\langle U\rangle$ is already at its correct steady-state value at $t=t_{W}$, its value keeps following the $T_{H} \rightarrow T_{C}$ equilibration curve and only later reverts back toward $k_{B} T_{W} / 2$. The observed behavior is known as the "anomalous Kovacs effect" [20]. In the Supplemental Material [40], we show that the very same behavior is observed when the initial state is prepared at $T_{C}$ and a heating Kovacs protocol is applied to reach $T_{W}$ (Figs. S3 and S4).

We resort to an analytical solution of Eq. (1), which bears qualitative similarity to the one developed in Ref. [46], to explain the measurements. As detailed in Sec. IV of the Supplemental Material [40], the equilibration of the potential energy can be described by the following equation:

$$
\begin{aligned}
\langle\tilde{U}\rangle & =1+a_{1} e^{-2 \lambda_{1} t}+a_{2} e^{-2 \lambda_{2} t}+a_{3} e^{-\Gamma t}, \\
\lambda_{1} & =\frac{1}{2}\left(\Gamma+\sqrt{\Gamma^{2}-4 \Omega_{0}^{2}}\right) \\
\lambda_{2} & =\frac{1}{2}\left(\Gamma-\sqrt{\Gamma^{2}-4 \Omega_{0}^{2}}\right)
\end{aligned}
$$

where $\tilde{U}$ is the potential energy normalized to its final value $k_{B} T_{f} / 2$ ( $T_{f}$ being the final temperature), $\lambda_{1}$ and $\lambda_{2}$ are the two poles of Eq. (1), and $\{a\}=\left\{a_{1}, a_{2}, a_{3}\right\}$ are coefficients that depend on the initial and final states and whose explicit expressions are given in Eqs. (S33) and (S34) in the Supplemental Material [40]. The equilibrations $T_{H} \rightarrow T_{C}$ and $T_{H} \rightarrow T_{W}$ can be computed in a straightforward fashion by substituting the values of the $\{a\}$ coefficients that correspond to the specific initial and final thermal states. For the case of the Kovacs protocol, we must compute the equilibration in three steps: (i) we use the equilibration $T_{H} \rightarrow T_{C}$ up to $t=t_{W}$, (ii) we use the instantaneous value $\langle U\rangle\left(t_{W}\right)$ and the final temperature $T_{W}$ to determine the new values of the $a$ coefficients, and (iii) compute the rest of the equilibration. The dashed, dash-dotted, and continuous lines in Fig. 2(b) represent the theoretical equilibrations computed from Eq. (2), which are in good agreement with the experiment. All quantities have been estimated independently and no fitting has been used in Fig. 2.

Deeper insight into the system's equilibration can be gained if we use the measured trajectories to estimate the position's probability distribution $\tilde{\rho}(x, t)$. The distributions for each of the three equilibration protocols are shown in Fig. 3. The position distributions exhibit a Gaussian shape at all instants of time. Specifically, during the Kovacs protocol, the distribution at the switch time $t_{W}$ is the same as in the steady state $t \rightarrow \infty$, i.e., Gaussians with the same variances. The fact that the distribution keeps narrowing for $t>t_{W}$ instead of remaining constant, however, implies that the particle's state is actually a nonequilibrium one. The solution to this apparent contradiction lies in the fact that Eq. (1) has two state variables, position $x$ and velocity $v$. The true state of the particle in phase space consists thus of a distribution $\rho(x, v, t)$ with two variables $(x, v)$ that changes in time. When measuring the position of the particle, we are averaging away the information about the velocity. In other words, the distributions in Fig. 3 are given by $\tilde{\rho}(x, t)=\int_{\mathbb{R}} d v \rho(x, v, t)$. These measurements do not describe the full state of the particle, and for this reason they cannot, in general, discern equilibrium states from nonequilibrium ones.

In order to reconstruct the evolution of the full distribution $\rho(x, v, t)$, analog formulas to Eq. (2) have been derived for the kinetic energy $\langle K\rangle$ and for the correlation $\langle x v\rangle$, see Eqs. (S47) and (S49) in the Supplemental Material [40]. Figure 4 shows the theoretical curves of 

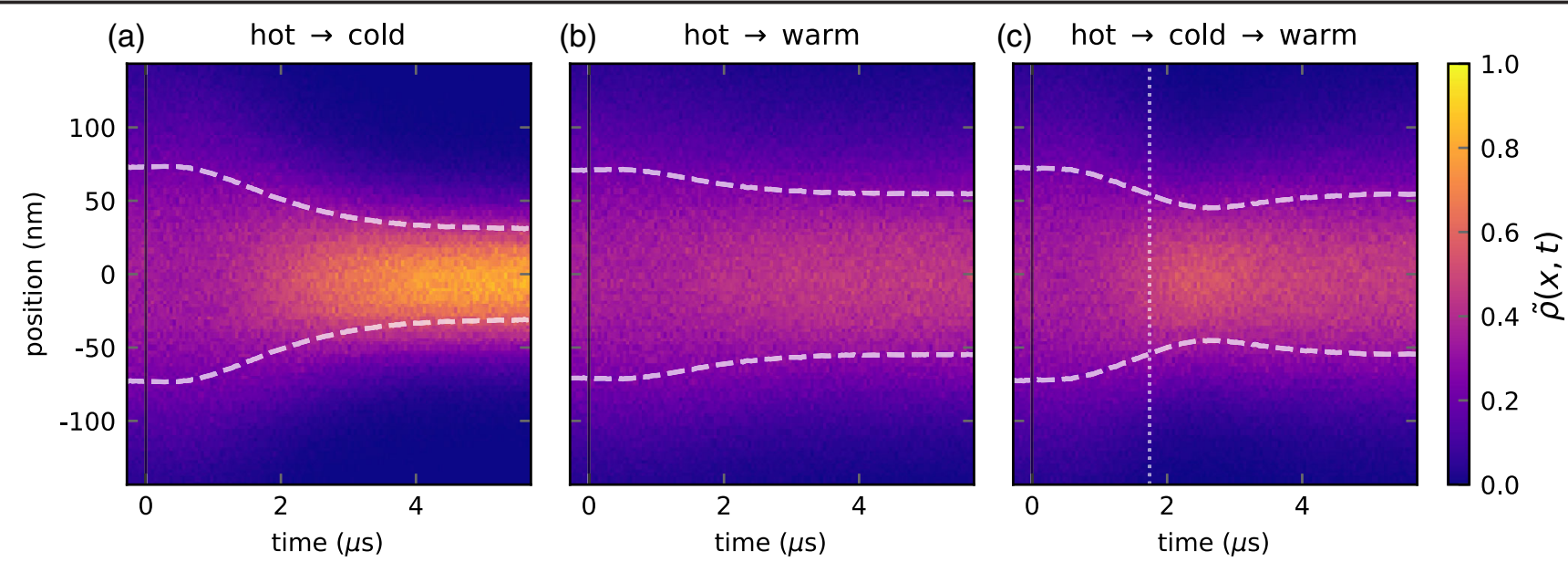

FIG. 3. (a) Measured position distribution $\tilde{\rho}(x, t)$ of the particle during the equilibration $T_{H} \rightarrow T_{C}$, (b) $T_{H} \rightarrow T_{W}$, (c) $T_{H} \rightarrow T_{C} \rightarrow T_{W}$ (Kovacs protocol). The vertical black line indicates the time $t=0$ where the effective temperature is first changed. (c) The vertical dotted white line shows the switch time $t=t_{W}$ between $T_{C}$ and $T_{W}$. The dashed white lines represent the standard deviations inferred from Fig. 2. At all times, the distributions are Gaussian. All the distributions are normalized to the maximum value of (a).

$\langle U\rangle,\langle K\rangle$, and $\langle E\rangle:=\langle U+K\rangle$ during the Kovacs protocol. We can see in Fig. 4 that these three energies follow different evolutions. As a result, the Kovacs protocol can be applied only to one of these thermodynamic quantities at a time. Remarkably, when the protocol is applied to the kinetic or to the total energy, a standard Kovacs effect is expected, as we show in Fig. S5 of the Supplemental Material [40]. It appears thus that a harmonic oscillator at intermediate damping values can exhibit either anomalous or standard Kovacs effect depending on the imposed protocol. The inset in Fig. 4 represents a snapshot of $\rho(x, v, t)$ taken at the Kovacs switch time $t=t_{W}$. The distribution is Gaussian and presents classical squeezing. In addition to this, there is a visible anticorrelation in $\langle x v\rangle$. This intermediate distribution is a nonequilibrium state, which explains the equilibrations in Fig. 2 despite the Gaussian distributions of Fig. 3. We refer to the Supplemental Material [40] for the time evolution of $\rho(x, v, t)$ for all $t \geq 0$.

In a deeply overdamped harmonic oscillator, one typically considers the dynamics of the particle as governed by a single timescale given by the cutoff frequency $\Omega_{c}=$ $\Omega_{0}^{2} / \Gamma$ [31,32]. However, a single timescale could not generate nonequilibrium states like the ones in Fig. 4, but rather only states that correspond to some intermediate temperature. A second, intrinsic timescale of the system is given by the damping coefficient $\Gamma$. The particle exhibits ballistic motion when its evolution is resolved within intervals on the order of $1 / \Gamma$, such that the overdamped approximation is never fully valid [47]. In the intermediate regime of our experiment, the ballistic and the cutoff timescales are close to each other. The result is that the equilibration of the potential energy is given by a superposition of three exponentials with time constants derived from both $\Gamma$ and $\Omega_{c}$. The superposition of these exponentials is the key to explain the measured Kovacs effect. In the case of the potential energy, the particle cannot respond instantaneously to the changes in temperature because of the ballistic motion over short times. This inertialike effect forces $\langle U\rangle$ during the Kovacs protocol to continue its decreasing trend even after the switch to $T_{W}$, generating thus an anomalous hump. Unlike $\langle U\rangle$, the changes of the kinetic energy are instantly affected by

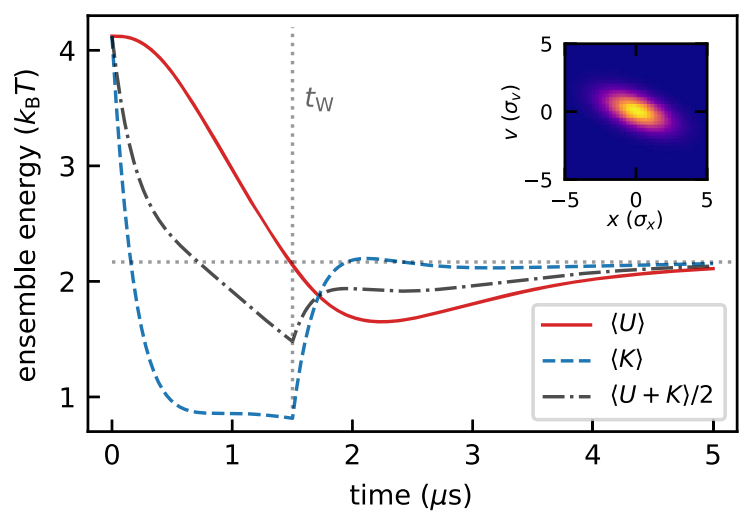

FIG. 4. Theoretical curves of potential $\langle U\rangle$, kinetic $\langle K\rangle$, and total $\langle E\rangle=\langle U+K\rangle$ energies during the Kovacs protocol for $\langle U\rangle$. At $t=0$ the effective temperature is switched from $T_{H}$ to $T_{C}$. At time $t_{W}$ (gray dotted vertical line), the effective temperature is switched to $T_{W}$. Since the thermal forces affect the velocity instantaneously, we see that the evolution of $\langle K\rangle$ (and consequently $\langle E\rangle$ ) presents kink points whenever the effective temperature is changed. In [40], we show that a Kovacs protocol applied to either the kinetic or the total energy would actually generate a standard Kovacs effect, i.e., the hump after $t_{W}$ points upward. Inset: phase space distribution $\rho(x, v, t)$ at $t=t_{W}$. The distribution is classically squeezed and has a nonzero $\langle x v\rangle$ correlation. The inset is normalized to its respective maximum and the same color bar as in Fig. 3 is used. 
the changes in temperature, which is the reason why Fig. 4 presents kink points in the evolution of $\langle K\rangle$. In [40], we prove that the Kovacs protocol applied to the kinetic energy presents a standard Kovacs effect. This can be understood as follows. At the switching time $t_{W}^{(K)}$ (different, in general, from $t_{W}$ ), the kinetic energy has the correct steady-state value, but the potential energy is higher. In order to reach equilibrium with $T_{W}$, the potential energy must first be converted to kinetic energy and then dissipated. It is this transfer from potential to kinetic energy that increases $\langle K\rangle$ at $t_{W}^{(K)}$ and gives rise to a standard Kovacs hump (see Fig. S5 in [40]).

In contrast, when the particle experiences low friction $\Gamma \ll \Omega_{0}$, the dynamics are underdamped and one finds a new separation of the effects of the two different timescales. On the one hand, the resonance frequency $\Omega_{0}$ induces coherent oscillations in the motion of the particle. On the other hand, the friction induces random fluctuations and dissipates the excess energy. For this underdamped regime, the first two exponentials of Eq. (2a) become vanishingly small and we recover an equilibration given by a single timescale, namely, $1 / \Gamma[40]$. The Kovacs effect in the harmonic oscillator is thus a result of the intermediate damping regime, which has been so far only marginally explored.

In conclusion, we have demonstrated that the energy equilibration of the harmonic oscillator can exhibit the Kovacs effect. This observation on one of the most widely used models in physics, the harmonic oscillator, highlights the generality and the importance of this phenomenon across fields. We emphasize that our experiments have been performed in the overdamped regime $\left(\Gamma^{2}>4 \Omega_{0}^{2}\right)$, but under a situation where the frequently used overdamped approximation, i.e., neglecting the first addend on the lhs term of Eq. (1), cannot be applied. This warns against the adoption of such an approximation under certain circumstances, because it may return erroneous conclusions and predictions, as has been previously observed in similar situations of low damping [48] or when the temperature of the system is allowed to evolve with time [49,50]. The accurate quantification of timescales in thermalization processes of Brownian systems is also required for the correct evaluation of performances and efficiencies in the implementation of thermodynamics processes and thermal machines at the microscale, the so-called stochastic thermodynamics [51-53]. Thermalization processes can also be engineered in order to achieve shorter relaxation times, although at a significant energetic cost [32]. Interestingly, the regime we explored here encompasses the critical damping, where the fastest relaxation naturally occurs and the efficiency of an engine can therefore be optimized $[27,50]$. An appropriate optimization needs to consider the occurrence of the Kovacs effect.

This research has been supported by European Union's Horizon 2020 Research and Innovation Programme under
Grant No. 863132 (iQLev) and by the Swiss National Science Foundation through Grant No. 200021L-169319. A. L. acknowledges financial support by the FEDER/Ministerio de Ciencia, Innovación y Universidades-Agencia Estatal de Investigación, under Grants No. MTM2017-84446-C2-2-R and No. PID2020-116567GB-C22. L. L. B. acknowledges financial support by the FEDER/Ministerio de Ciencia, Innovación y Universidades-Agencia Estatal de Investigación, under Grants No. MTM2017-84446-C2-2-R and No. PID2020-112796RB-C22, the Madrid Government (Comunidad de Madrid-Spain) under the Multiannual Agreement with UC3M in the line of Excellence of University Professors (EPUC3M23), and in the context of the V PRICIT (Regional Programme of Research and Technological Innovation). R. A. R. acknowledges financial support from FEDER/Junta de Andalucía-Consejería de Economía y Conocimiento/Projects No. C-FQM-410UGR18 and No. P18-FR-3583.

*rul@ugr.es

$\dagger$ www.photonics.ethz.ch

[1] N. C. Keim, J. D. Paulsen, Z. Zeravcic, S. Sastry, and S. R. Nagel, Rev. Mod. Phys. 91, 035002 (2019).

[2] L. Berthier and J.-P. Bouchaud, Phys. Rev. B 66, 054404 (2002).

[3] M. Baity-Jesi et al. (Janus Collaboration), Phys. Rev. Lett. 120, 267203 (2018).

[4] G. F. Rodriguez, G. G. Kenning, and R. Orbach, Phys. Rev. Lett. 91, 037203 (2003).

[5] L. M. Janssen, A. Kaiser, and H. Löwen, Sci. Rep. 7, 5667 (2017).

[6] C. L. Struik, Physical Aging in Amorphous Polymers and Other Materials (Elsevier, Amsterdam, 1980).

[7] E. B. Mpemba and D. G. Osborne, Phys. Educ. 4, 172 (1969).

[8] Z. Lu and O. Raz, Proc. Natl. Acad. Sci. U.S.A. 114, 5083 (2017).

[9] I. Klich, O. Raz, O. Hirschberg, and M. Vucelja, Phys. Rev. X 9, 021060 (2019).

[10] A. Lasanta, F. Vega Reyes, A. Prados, and A. Santos, Phys. Rev. Lett. 119, 148001 (2017).

[11] A. Torrente, M. A. López-Castaño, A. Lasanta, F. V. Reyes, A. Prados, and A. Santos, Phys. Rev. E 99, 060901(R) (2019).

[12] M. Baity-Jesi et al. (Janus Collaboration), Proc. Natl. Acad. Sci. U.S.A. 116, 15350 (2019).

[13] A. Gijón, A. Lasanta, and E. R. Hernández, Phys. Rev. E 100, 032103 (2019).

[14] A. Nava and M. Fabrizio, Phys. Rev. B 100, 125102 (2019).

[15] F. Carollo, A. Lasanta, and I. Lesanovsky, Phys. Rev. Lett. 127, 060401 (2021).

[16] A. J. Kovacs, Fortschr. Hochpolym. Forsch. 3/3, 394 (1964).

[17] A. J. Kovacs, J. J. Aklonis, J. M. Hutchinson, and A. R. Ramos, J. Polym. Sci. B 17, 1097 (1979).

[18] G. Aquino, L. Leuzzi, and T. M. Nieuwenhuizen, Phys. Rev. B 73, 094205 (2006).

[19] A. Lasanta, F. Vega Reyes, A. Prados, and A. Santos, New J. Phys. 21, 033042 (2019). 
[20] A. Prados and E. Trizac, Phys. Rev. Lett. 112, 198001 (2014).

[21] R. Kürsten, V. Sushkov, and T. Ihle, Phys. Rev. Lett. 119, 188001 (2017).

[22] S. Mossa and F. Sciortino, Phys. Rev. Lett. 92, 045504 (2004).

[23] C. A. Plata and A. Prados, Entropy 19, 539 (2017).

[24] A. Parravicini, J. A. Agranat, C. Conti, and E. Del Re, Appl. Phys. Lett. 101, 111104 (2012).

[25] C. Josserand, V. A. Tkachenko, D. M. Mueth, and H. M. Jaegger, Phys. Rev. Lett. 85, 3632 (2000).

[26] J. Gieseler, R. Quidant, C. Dellago, and L. Novotny, Nat. Nanotechnol. 9, 358 (2014).

[27] I. A. Martínez, É. Roldán, L. Dinis, D. Petrov, J. M. Parrondo, and R. A. Rica, Nat. Phys. 12, 67 (2016).

[28] J. X. Zhu, D. J. Durian, J. Müller, D. A. Weitz, and D. J. Pine, Phys. Rev. Lett. 68, 2559 (1992).

[29] B. Lukić, S. Jeney, C. Tischer, A. J. Kulik, L. Forró, and E.-L. Florin, Phys. Rev. Lett. 95, 160601 (2005).

[30] T. Franosch, M. Grimm, M. Belushkin, F. M. Mor, G. Foffi, L. Forró, and S. Jeney, Nature (London) 478, 85 (2011).

[31] A. Kumar and J. Bechhoefer, Nature (London) 584, 64 (2020).

[32] I. A. Martínez, A. Petrosyan, D. Guéry-Odelin, E. Trizac, and S. Ciliberto, Nat. Phys. 12, 843 (2016).

[33] M. Chupeau, S. Ciliberto, D. Guéry-Odelin, and E. Trizac, New J. Phys. 20, 075003 (2018).

[34] L. Rondin, J. Gieseler, F. Ricci, R. Quidant, C. Dellago, and L. Novotny, Nat. Nanotechnol. 12, 1130 (2017).

[35] J. Gieseler, B. Deutsch, R. Quidant, and L. Novotny, Phys. Rev. Lett. 109, 103603 (2012).

[36] E. Hebestreit, M. Frimmer, R. Reimann, C. Dellago, F. Ricci, and L. Novotny, Rev. Sci. Instrum. 89, 033111 (2018).

[37] I. A. Martínez, E. Roldán, J. M. R. Parrondo, and D. Petrov, Phys. Rev. E 87, 032159 (2013).
[38] M. Frimmer, K. Luszcz, S. Ferreiro, V. Jain, E. Hebestreit, and L. Novotny, Phys. Rev. A 95, 061801(R) (2017).

[39] R. Kubo, Rep. Prog. Phys. 29, 255 (1966).

[40] See Supplemental Material, which includes Refs. [41,42], at http://link.aps.org/supplemental/10.1103/PhysRevLett.127 .130603 for technical details on the experimental setup, calibration, and the detailed theoretical treatment of the problem, including parallel derivations of the Kovacs effect (Langevin and Focker-Planck formalisms). An animation of the evolution of $\rho(x, v, t)$ during the Kovacs protocol is provided, along with additional experimental results.

[41] S. A. Beresnev, V. G. Chernyak, and G. A. Fomyagin, J. Fluid Mech. 219, 405 (1990).

[42] H. Risken and T. Frank, The Fokker-Planck Equation: Methods of Solutions and Applications, Springer Series in Synergetics (Springer, New York, 1996).

[43] F. Tebbenjohanns, M. Frimmer, A. Militaru, V. Jain, and L. Novotny, Phys. Rev. Lett. 122, 223601 (2019).

[44] E. M. Bertin, J.P. Bouchaud, J. M. Drouffe, and C. Godrèche, J. Phys. A 36, 10701 (2003).

[45] A. Prados and J. J. Brey, J. Stat. Mech. (2010) P02009.

[46] S. F. Nørrelykke and H. Flyvbjerg, Phys. Rev. E 83, 041103 (2011).

[47] T. Li, S. Kheifets, D. Medellin, and M. G. Raizen, Science 328, 1673 (2010).

[48] R. Pan, T. M. Hoang, Z. Fei, T. Qiu, J. Ahn, T. Li, and H. T. Quan, Phys. Rev. E 98, 052105 (2018).

[49] É. Roldán, I. A. Martínez, L. Dinis, and R. A. Rica, Appl. Phys. Lett. 104, 234103 (2014).

[50] I. A. Martínez, E. Roldán, L. Dinis, D. Petrov, and R. A. Rica, Phys. Rev. Lett. 114, 120601 (2015).

[51] U. Seifert, Rep. Prog. Phys. 75, 126001 (2012).

[52] I. A. Martínez, É. Roldán, L. Dinis, and R. A. Rica, Soft Matter 13, 22 (2017).

[53] J. Gieseler and J. Millen, Entropy 20, 326 (2018). 